\title{
Rare Case of Servelle Martorelle Syndrome
}

\author{
Bhatnagar A, Deshpande M
}

Dept of Plastic Surgery

SGPGIMS, Lucknow, India

Corresponding Author

Ankur Bhatnagar

Dept of Plastic Surgery

SGPGIMS, Lucknow, India

Email: bhatnagarankur2000@yahoo.com

\begin{abstract}
Servelle Martorelle Syndrome is a congenital vascular malformation associated with soft tissue hypertrophy and bony hypoplasia. This rarely involves whole of an extremity, with involvement of part of limbs reported in literature. We present a case of a twelve year boy who presented to the Department of Plastic Surgery SGPGIMS in April 2011 , with history of circumferential soft tissue hypertrophy involving whole of left upper limb, scapular region and axilla since birth. The entire left upper limb length was lesser than the right upper limb. Hence this is a very rare case of Servelle Martorelle Syndrome having extensive limb involvement at a very young age. Highlighted is the role of conservative treatment and close follow-up to understand the natural history of the diseases, with prompt treatment of complications.
\end{abstract}

\section{Citation}

Bhatnagar A, Deshpande M. Rare Case of Servelle Martorelle Syndrome. Kathmandu Univ Med J 2012;10(4):91-94.

\section{KEY WORDS}

Angio-osteohypotrophic angiodysplasia, venous malformation

\section{INTRODUCTION}

Angio-osteohypotrophic syndrome known as ServelleMartorelle angiodysplasia describes the association of venous and rarely, arterial malformations with skeletal abnormalities. ${ }^{1,2}$ The ectasia and dilatation of the superficial veins result in a monstrous deformity of the extremity. Intraosseous vascular malformations may lead to hypotrophy of bone with destruction of spongiosa and cortical bone, resulting in shortening of the limb, cystic changes in medullary cavity and joint destruction. ${ }^{3,4}$

This syndrome is frequently confused with KlippelTrenaunay, Parkes-Weber and Blue rubber bleb nevus syndromes, which present with limb and bone hypertrophy. $\mathrm{MRI}$ is the best imaging method for diagnosis. ${ }^{5}$

Adequate radiological investigations with corroborative clinical findings are crucial to establish correct diagnosis. The prognosis of this disorder is uncertain. Therapy is predominantly conservative. In the presence of aneurysmal complications or severe shunting, surgery may be indicated. Servelle-Martorell syndrome especially involving the entire limb, axilla and chest wall has been reported rarely in the literature. ${ }^{6}$ There is very little information about the genetic basis of this syndrome. ${ }^{7}$

\section{CASE REPORT}

A twelve year old boy presented to the Department of Plastic Surgery, SGPGIMS in April 2011 with history of circumferential irregular soft tissue hypertrophy involving whole of left upper limb, scapular region and axilla since birth. The swelling initially involved the forearm and hand but gradually extended both in size and extent with age. The pain and swelling were worse when the limb was lowered with complaints of night cramps for past two years. Patient also complained that for last four years he has had multiple episodes of pain in the upper limb which has responded to rest, elevation, analgesic and antibiotics.

Examination showed that the entire upper limb has multiple separate swollen areas over the whole of the arm and shoulder girdle of different sizes(Fig 1). They were soft and compressible, and significantly decreased in size with elevation .The left arm was shorter than the right by $5 \mathrm{~cm}$ and this reduction in length was due to overall shortening rather than localized shortening within a particular section of the limb(Fig 2).

The arm muscles and muscles of the limb girdle were atrophic. The soft tissue swelling had characteristic of venous ecstasies. There was no significant family history. 
The whole left upper limb had dilated engorged veins extending up to left half of both anterior and posterior aspect of chest wall. Other parts of the body showed no abnormalities. The peripheral pulses were palpable with equal volume on both sides.

The arm, forearm and hand bones were hypo plastic and tender. There was no sensory deficit. The muscle strength of the right upper limb was grade III to IV. No bruits or thrills were found. Left upper limb felt warmer than right. There was adequate range of motion at the elbow and shoulder, with severe restriction of flexion and poor grip due excess soft tissue in the palm (Fig 3). The cardiovascular examination and other systemic examination were normal.

Blood investigation revealed a normal blood picture. Radiographs showed multiple soft tissue swellings and hypotrophy of the bones of left upper limb. There were multiple well-defined radio-opaque lesions consistent with phleboliths in the affected upper limb and periscapular region (Fig 4, 5).

Musculoskeletal ultrasound showed multiple dilated tortuous, anechoic lesions involving the upper limb and periscapular region. Echogenic lesions with shadowing suggestive of phleboliths were seen inside the anechoic lesions. The forearm muscles were thinned and atrophic.

Color Doppler study showed features suggestive of extensive low-flow venous malformation.

An MRI study showed multiple dilated veins in the superficial aspect of the right upper limb extending into the axilla and the periscapular region (Fig.6). The muscles of the limb were replaced by an abnormal vascular tissue and most of the muscles were atrophic. The triceps, biceps and deltoid were partially involved. The subcutaneous tissues along with the pectoral and periscapular musculature were grossly involved with muscular atrophy ( Fig.7). The arteries were grossly normal. Further diagnostic procedures were declined by the parents due to financial constraints.

A detailed discussion was done with the parents regarding the cause, nature and prognosis of the diseases. All potential complications were explained to them. In view of the extensive lesion patient was managed conservatively.

The child was given custom made graduated compression garments. The child has been instructed to use them as much as possible. Garments are removed at night only with instructions to have limb elevated during sleep. Compression therapy has helped to diminish the symptoms of venous insufficiency. The child has been on compression garments for last six months with marked decrease in pain and tenderness and absence of night cramps. There is marked cosmetic improvement due to the pressure garment and child is able to wear normal size clothes for his age. The patient has not had any complications such as venous thrombosis, consumption coagulopathy, cellulitis or bleeding while on compression garment.

\section{DISCUSSION}

Congenital Vascular malformations (CVM) as a group of lesions have been difficult not only to treat but also to classify. This is mainly due to varied nature of the diseases and requirement of a multi disciplinary team to treat these patients adequately, where different specialties tend to classify the vascular malformations differently.

An important contribution in this regard was made by Mulliken whose pioneering efforts have led to the development of a workable system of classification for CVM. This new classification is known as the Hamburg classification. $^{2}$ This classification was introduced after reappraisal of the older terminology and has become the standard system for contemporary classification, based on anatomical, pathophysiological, histological, hemodynamic status and embryological criterion. ${ }^{8}$

However the name based eponyms have been used extensively to describe and classify many complex vascular malformations. Most of these vascular malformations have extra vascular e.g. cutaneous and bone involvement. Although these eponyms fail to provide essential information regarding etiology, anatomy and Pathophysiology, each of these syndromes has an important historical significance. They provide a clinical description of the primary vascular lesion along with its secondary non vascular lesions and clinical findings (soft tissue swellings, bone growth discrepancy, cutaneous manifestations). Thus these eponyms are still useful for certain combined complex vascular malformations. ${ }^{8}$

Langer demonstrated that differentiation of these syndromes is possible by taking standard X-rays of the extremities Weber syndrome: bony lesions with limb hypertrophy, Klippel-Trenaunay syndrome: bony lesions do not accompany lengthening, Servelle-Martorell syndrome: bony lesions with limb hyportrophy. ${ }^{3}$ The history and clinical examination along with investigations allowed us to label this as an osteo hypotrophic syndrome known as SERVELLE MARTORELLE SYNDROME which is a phlebectactic osteohypoplastic angio dysplasia.

Our case is a rare variant of this less reported vascular syndrome. There is a progressively increasing extensive involvement of the entire upper limb, axilla and shoulder girdle while in majority of the cases the involvement is confined to a single or small part of the limb. Secondly this is first case of such extensive involvement at a very young age. A meticulous follow up of this case will help us in getting a better understanding of the natural history of the diseases, as well as understanding the long term prognosis and management of various complications.

Various theories have been postulated regarding the pathogenesis of osteohypotrophy, however mechanical compression of the vascular channels leading to reduced bone circulation due to hypotrophy of the feeding arteries appears to be the most plausible reason. ${ }^{9}$ 


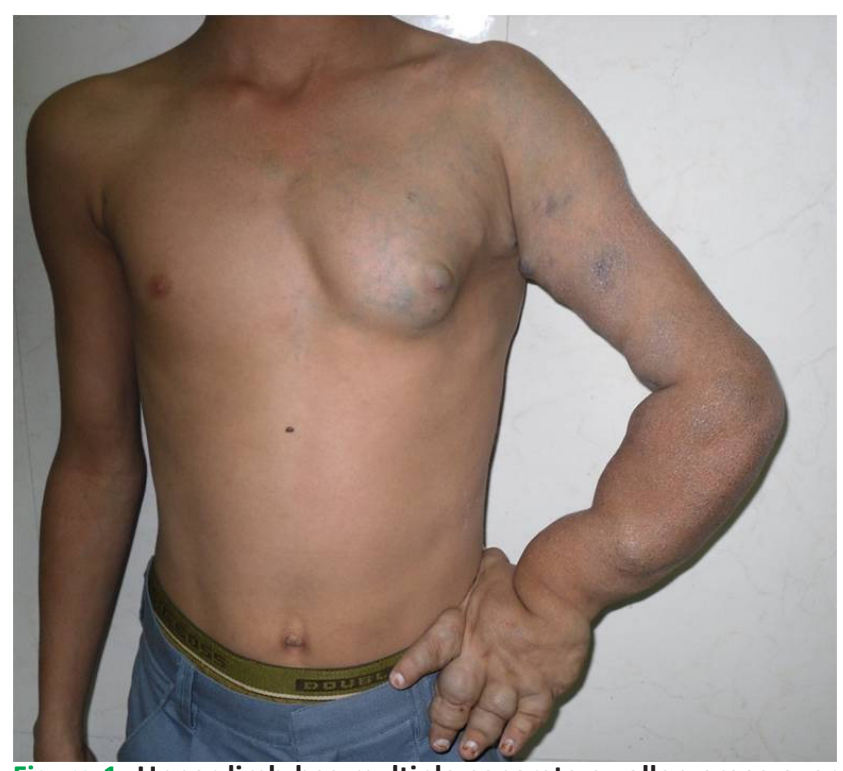

Figure 1. Upper limb has multiple separate swollen areas over the whole of the arm and shoulder girdle.

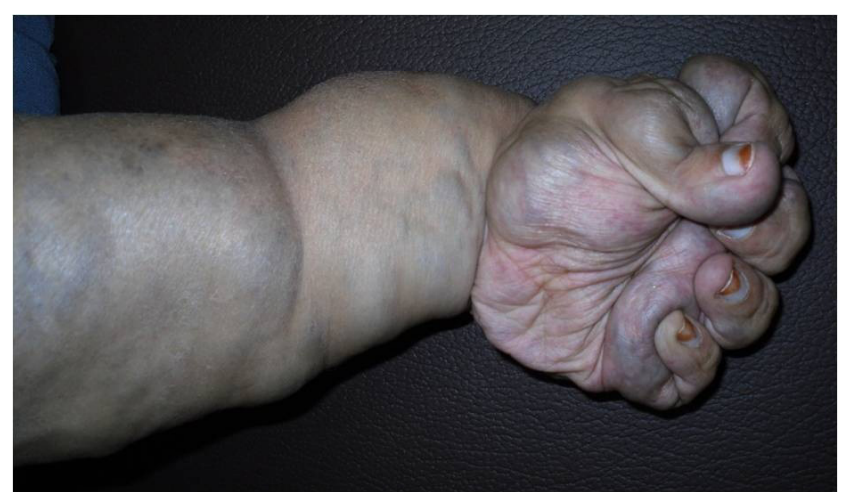

Figure 3. Severe restriction of flexion and poor grip due excess soft tissue in the palm.

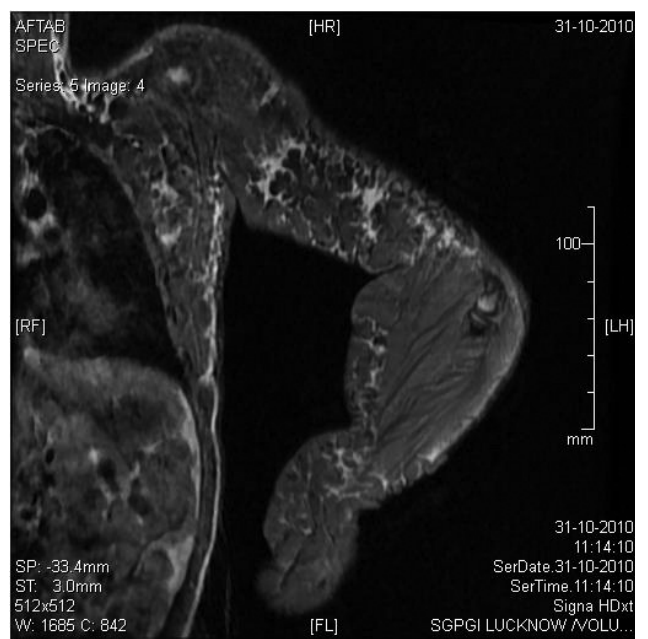

Figure 6. MRI showing multiple dilated veins in the superficial aspect of the right upper limb extending into the axilla and the periscapular region.

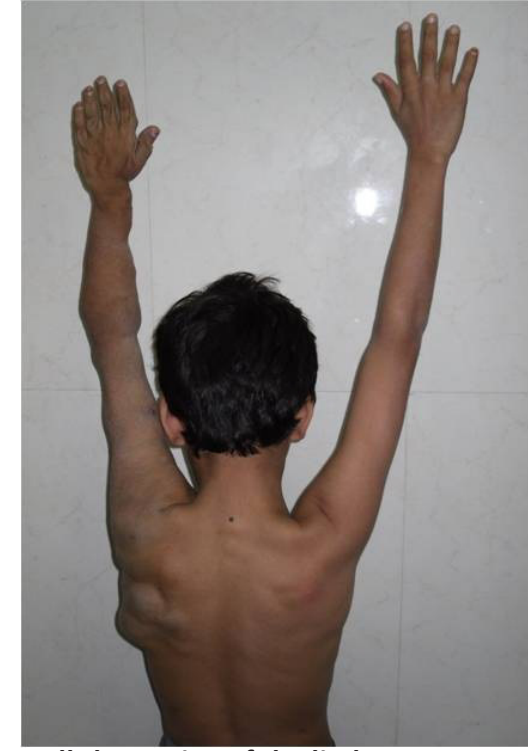

Figure 2. Overall shortening of the limb.

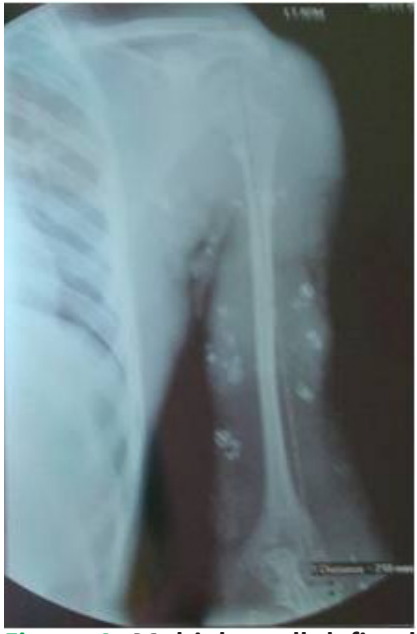

Figure 4. Multiple well-defined radio-opaque lesions in arm consistent with phleboliths

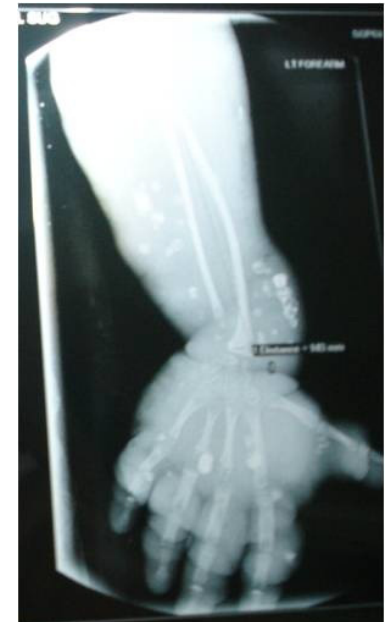

Figure 5. Multiple radioopaque lesions in forearm and hand consistent with phleboliths.

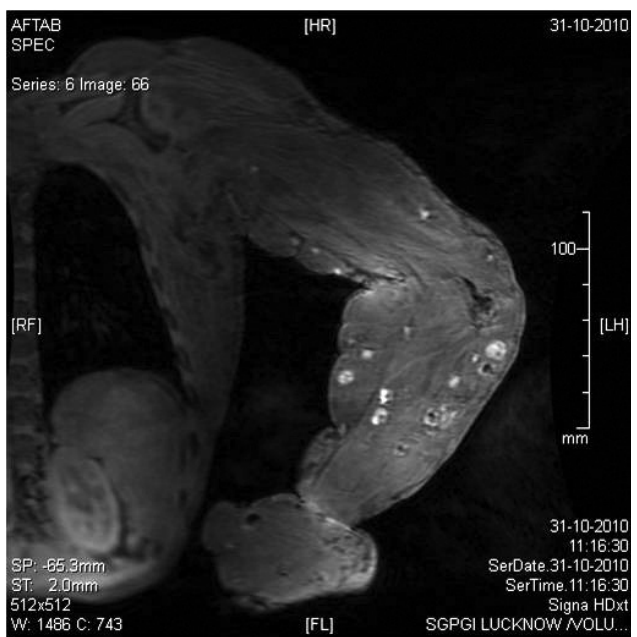

Figure 7. MRI showing subcutaneous tissue involvement with gross muscular atrophy and phleboliths. 
In view of such extensive involvement at a very young age we have decided to put the child on conservative treatment after due consultation with the parents. ${ }^{10}$ The child has been on custom made graduated compression stockings for last one year. There has been a marked improvement in pain and limb tenderness. There has been no episode of night cramps after using compression garments. Child has been advised limb elevation while sleeping. There has been no episode of fever following compression therapy. Compression garment also provides protection against accidental injury to the skin which may lead to troublesome hemorrhage and ulceration, which are difficult to treat.

The biggest advantage of compression garments according to the child has been the ability to wear clothes appropriate to his age and a better grip and pinch of the hand which however is far from normal.

For limb length discrepancy, various orthopedic procedures have been advocated both in the growing age group and after skeletal maturity. ${ }^{10}$ However in our patient due to poor bone stock and extensive soft tissue lesion no such procedure could be contemplated. Compression garment does not have any effect on the overall limb length. No evidence of any distal vascular insufficiency was seen.

It is our firm belief that surgical intervention in such extensive lesions is of no use and should be best avoided.

Other measures such as sclerotherapy can only help localized lesions and were not tried in our case. These are left for management of emergencies and complications only. Arterial embolization of this predominantly venous malformation would lead vascular insufficiency hence not tried.

Regarding the cytogenetics of this lesion much less is known as compared to other commonly reported osteohypotrophy vascular syndromes. Reciprocal translocations $t(5 ; 11)$ and $\mathrm{t}(8 ; 14)$ and ring chromosome of 18 are reported ,however no genetic defect has been identified yet. ${ }^{7}$

\section{CONCLUSION}

SMS with such extensive limb involvement at a very young age makes it rarest of the rare cases. Servelle Martorelle Syndrome is an uncommonly reported entity. One of the reasons for this we feel may be due to the fact that many of these patients are labeled as cases of Klippel Trenaunay syndrome. However a good history and clinical examination and basic investigation are enough to differentiate both these conditions. MRI is the most useful in the diagnosis of this problem.

Surgery and other invasive procedures should be reserved for localized lesions or in the management of complications. Conservative treatment in form of compression garment, proper patient counseling and strict follow up with prompt management of any complications should be mainstay of treatment.

Such cases with extensive involvement at a young age if followed well, will help us to ascertain the natural history of the diseases, about which less is known. It will also throw light on the nature of bone development during growth phase and after skeletal maturity .This patient with close follow up will help us understand the long term impact of conservative treatment on the progression of the diseases process, especially the bone hypotrophy and limb length discrepancy.

Hence we feel that regular, careful clinical examination will help in avoiding the various complications associated with the syndrome and conservative treatment in the form of compression garments should remain the mainstay of the treatment.

\section{REFERENCES}

1. Enjolras $\mathrm{O}$, Mulliken JB . Vascular malformations Textbook of Pediatric Dermatology.Oxford: Blackwell Science; 2000.

2. Enjolras $\mathrm{O}$, Mulliken JB . Vascular tumours and vascular malformations (new issues). Advances in Dermatology 1998; 13:375-423.

3. Langer $\mathrm{M}$, Langer R. Radiologic aspects of the congenital arteriovenous malformations, Klippel-Trenaunay type and Servelle-Martorell type. Rofo. 1982; 136:577-582.

4. Mattassi R, Vaghi M.Vascular Bone Syndrome -Angio Osteodystrophy :Current Concept. Phlebology. 2007;22(6) :287-290.

5. Lee BB, Laredo J, Lee SJ, Huh SH. Congenital vascular malformations General Diagnostic Principals. Phlebology. 2007;22(6): 253-257.

6. Karuppal R, Raman RV .Servelle Martorelle Syndrome with Extensive upper limb involvement: A case report.Journal of Medical Case Reports. 2008; 2: 142.

7. Phadke SR. Klippel Trenaunay syndrome. Atlas Genet Cytogenet Oncol Haematol. Jan 2008.

8. Lee BB, Laredo J, Kim YM, Neville R, Huh SH. Terminology and Classification of Congenital vascular malformations. Phlebology .2007;22(6): 249-252.

9. Belov St. Hemodynamic pathogenesis of vascular bone syndromes in congenital vascular defects. Int Angiol. 1990; 9:155-61.

10. Angel C, Yngve D, Murillo C, Hendrick E, Adegboyega P, Swischuk L.Surgical treatment of vascular malformations of the extremities in children and adolescents. Pediatr Surg Int. 2002; 18:213-217. 